\title{
GESTIÓN ECONÓMICAMENTE OPTIMIZADA DEL AGUA DE RIEGO EN UNA EXPLOTACIÓN AGRÍCOLA MEDIANTE EL MODELO "MOPECO"
}

\author{
Martínez, A.(1)(P), Martínez-Romero, A.(2), López-Mata, E.(2), Tarjuelo, J.M.(2), Pardo, J.J.
}

(1), Domínguez, A.(2)

1 Ingeniero Agrónomo, Doctorando, Universidad de Castilla-La Mancha. Antonio.Martinez26@alu.uclm.es

${ }^{2}$ Investigador, Centro Regional de Estudios del Agua (CREA), Universidad de Castilla-La Mancha, Ctra. de Las Peñas, km 3.2 , 02071 Albacete (Spain), Angel.MRomero@uclm.es; Eulogio.Lopez@uclm.es; Jose.Tarjuelo@uclm.es; JoseJesus.Pardo@uclm.es; Alfonso.Dominguez@uclm.es

\section{1- Introducción y Objetivos}

Es un hecho que los recursos hídricos para la agricultura en Castilla-La Mancha son escasos y el coste de utilización de éstos cada vez más elevado, por lo que los márgenes brutos obtenidos por los agricultores están llegando a los límites de la viabilidad económica.

Desde hace varios años se viene desarrollando en el CREA el modelo MOPECo (modelo de optimización económica del agua de riego) (Ortega et al., 2004), cuya finalidad es maximizar el margen bruto de beneficio de las explotaciones de regadío mediante una mayor eficiencia en el uso del agua de riego. Para una superficie regable y una cantidad de agua de riego disponible limitada, este modelo determina la superficie y la cantidad de agua de riego a aplicar a cada cultivo, estableciendo estrategias de riego deficitario optimizado por etapas (ORDI) (Domínguez et al., 2012) que maximizan la eficiencia en el uso del agua de riego en términos de kilogramo de cosecha por $\mathrm{mm}$ de agua aplicada.

En explotaciones que disponen de una cantidad limitada de agua de riego, parte de la explotación puede quedar sin cultivar (salvo con cultivos de secano) si se introducen en la rotación cultivos de altas necesidades, por lo que la aplicación de técnicas de riego deficitario permitiría incrementar el porcentaje de superficie regable cultivada de la explotación, pudiendo incrementar el margen bruto total obtenido.

El objetivo de este trabajo es aplicar MOPECO en una explotación de regadío real, y comparar los resultados económicos obtenidos con el manejo tradicional del riego de la misma, es decir, aplicando a los cultivos la cantidad de agua suficiente para cubrir sus necesidades, para una distribución de tres cultivos: cebada, cebolla y maíz.

\section{2- Materiales y métodos}

El trabajo de campo se ha realizado en una explotación de regadío ubicada en la provincia de Albacete. Los cultivos a introducir en la distribución que se pretende optimizar son: maíz C700, cebolla grano y cebada.

En todos los cultivos se han realizado dos tipos diferentes de estrategias de riego (No deficitario y ORDI) con cuatro repeticiones durante la campaña 2015. En el No deficitario se ha tratado de cubrir el $100 \%$ de las necesidades hídricas de los cultivos, 
calculadas según la metodología FAO (Allen et al., 1998) y en el segundo tratamiento el modelo MOPECO ha determinado el nivel de déficit global y por etapa a aplicar a cada cultivo, así como el calendario de riegos deficitarios, basado en la metodología ORDI, capaz de lograr los objetivos de déficit. La metodología ORDI (optimized regulated deficit irrigation) determina los niveles de déficit óptimos a aplicar (relación ETa/ETm) en cada etapa de desarrollo de los cultivos herbáceos en función del nivel de déficit global que se desee alcanzar. Para el control efectivo del contenido de humedad del suelo, se han utilizado sensores de tipo tensiómetro (Watermark) y volumétrico (Enviroscan), cuyas lecturas se han comparado con las estimadas por el modelo MOPECO. Los datos climáticos utilizados pertenecen a la estación "El Sanchón" (Casas de Fernando Alonso, Cuenca), situada próxima a la explotación y perteneciente a la red de estaciones SIAR.

Una vez obtenidos los valores de cosecha de los cultivos para ambos tratamientos, se han calculado y comparado los márgenes brutos reales obtenidos con las dos estrategias para un escenario económico (Tabla 1). Así, se ha considerado una explotación de 100 ha regables con una dotación de $5.000 \mathrm{~m}^{3} / \mathrm{ha}$. Con el fin de lograr una distribución de cultivos lo más realista posible en términos de cumplimiento de la normativa establecida por la PAC, así como los condicionantes agronómicos que recomiendan realizar una adecuada rotación de cultivos, se ha establecido que la superficie mínima que se debe dedicar a cada cultivo es de 20 ha.

Tabla 1. Precios de venta, costes de cultivo y coste del agua de riego.

Coste del agua $\left(€ / \mathrm{m}^{3}\right): 0,12 €$

\begin{tabular}{cccc}
\multicolumn{2}{c}{ Precio venta cosecha $(€ / \mathbf{K g})$} & Paja $(€ / \mathbf{K g})$ & Coste cultivo $(€ /$ ha \\
\hline Cebada & 0,16 & 0,04 & 580,20 \\
Cebolla & 0,11 & & $2.924,90$ \\
Maíz & 0,17 & 0,02 & $1.751,00$
\end{tabular}

\section{3- Resultados y discusión.}

Mopeco determinó como niveles globales de déficit: $70 \%$ de la ETm para cebada, y $90 \%$ de la ETm para cebolla y maíz. Los niveles de déficit (entendidos como relaciones ETa/ETm) a aplicar en cada una de las etapas de desarrollo de estos tres cultivos según ORDI aparecen recogidos en la Tabla 2. Bajo condiciones reales de cultivo, en ocasiones no es posible alcanzar las relaciones ETa/ETm objetivo como consecuencia de la distribución de las lluvias y los condicionantes que afectan al manejo de los sistemas de riego (Tabla 2).

Tabla 2. Relaciones ETa/ETm objetivo por etapas y reales alcanzadas.

TRATAMIENTOS SIN DÉFICIT

\begin{tabular}{|c|c|c|c|c|c|c|}
\hline & \multicolumn{2}{|c|}{ Cebada } & \multicolumn{2}{|c|}{ Cebolla } & \multicolumn{2}{|c|}{ Maíz } \\
\hline $\begin{array}{l}\text { Etapas } \\
\text { fenológicas }\end{array}$ & $\begin{array}{l}\text { ETa/ETm } \\
\text { real }\end{array}$ & $\begin{array}{c}\text { ETa/ETm } \\
\text { objetivo }\end{array}$ & $\begin{array}{l}\text { ETa/ETm } \\
\text { real }\end{array}$ & $\begin{array}{c}\text { ETa/ETm } \\
\text { objetivo }\end{array}$ & $\begin{array}{l}\text { ETa/ETm } \\
\text { real }\end{array}$ & $\begin{array}{c}\text { ETa/ETm } \\
\text { objetivo }\end{array}$ \\
\hline Establecimiento & 1,00 & 1,00 & 0,99 & 1,00 & 1,00 & 1,00 \\
\hline $\begin{array}{l}\text { Desarrollo } \\
\text { vegetativo }\end{array}$ & 1,00 & 1,00 & 0,95 & 1,00 & 1,00 & 1,00 \\
\hline Floración & 0,97 & 1,00 & --- & --- & 0,99 & 1,00 \\
\hline $\begin{array}{l}\text { Desarrollo del } \\
\text { grano } \\
\text { /bulbificación }\end{array}$ & 0,95 & 1,00 & 0,95 & 1,00 & 1,00 & 1,00 \\
\hline Maduración & 0,96 & 1,00 & 0,94 & 1,00 & 0,99 & 1,00 \\
\hline
\end{tabular}


TRATAMIENTOS ORDI

\begin{tabular}{lcccccc} 
& \multicolumn{2}{c}{ Cebada } & \multicolumn{2}{c}{ Cebolla } & \multicolumn{2}{c}{ Maíz } \\
\hline Etapas & $\begin{array}{c}\text { ETa/Etm } \\
\text { real }\end{array}$ & $\begin{array}{c}\text { ETa/Etm } \\
\text { objetivo }\end{array}$ & $\begin{array}{c}\text { ETa/Etm } \\
\text { real }\end{array}$ & $\begin{array}{c}\text { ETa/Etm } \\
\text { objetivo }\end{array}$ & $\begin{array}{c}\text { ETa/Etm } \\
\text { real }\end{array}$ & $\begin{array}{c}\text { ETa/Etm } \\
\text { objetivo }\end{array}$ \\
Establecimiento & 1,00 & 0,80 & 0,96 & 0,88 & 1,00 & 0,80 \\
Desarrollo & 0,75 & 0,55 & 0,89 & 0,92 & 0,67 & 0,70 \\
vegetativo & 0,74 & 0,80 & --- & --- & 0,98 & 1,00 \\
Floración & 0,54 & 0,79 & 0,95 & 1,00 & 1,00 & 0,99 \\
Desarrollo del & 0,55 & 0,54 & 0,68 & 0,65 & 0,85 & 0,94 \\
grano /bulbificación & $\mathbf{0 , 7 0}$ & $\mathbf{0 , 7 0}$ & $\mathbf{0 , 9 0}$ & $\mathbf{0 , 9 0}$ & $\mathbf{0 , 9 2}$ & $\mathbf{0 , 9 0}$ \\
Maduración & & & & & &
\end{tabular}

Las figuras 1 y 2 muestran, a modo de ejemplo para el maíz, la evolución del contenido de humedad del suelo teórico según el modelo y la distribución de los riegos. En el caso de los tratamientos no deficitarios (Fig. 1) el objetivo fue que el contenido de humedad del suelo (línea negra), se mantuviera por encima del nivel de agotamiento disponible (línea gris), con el fin de que el cultivo no entrara en estrés. La Figura 2, muestra como durante las etapas de desarrollo vegetativo y maduración, el contenido de humedad del suelo estuvo por debajo del nivel de agotamiento disponible hasta alcanzar la relación ETa/ETm objetivo de estas etapas (línea negra rayada) (Tabla 2). Las líneas verticales indican el momento en el que se produce el cambio de etapa.

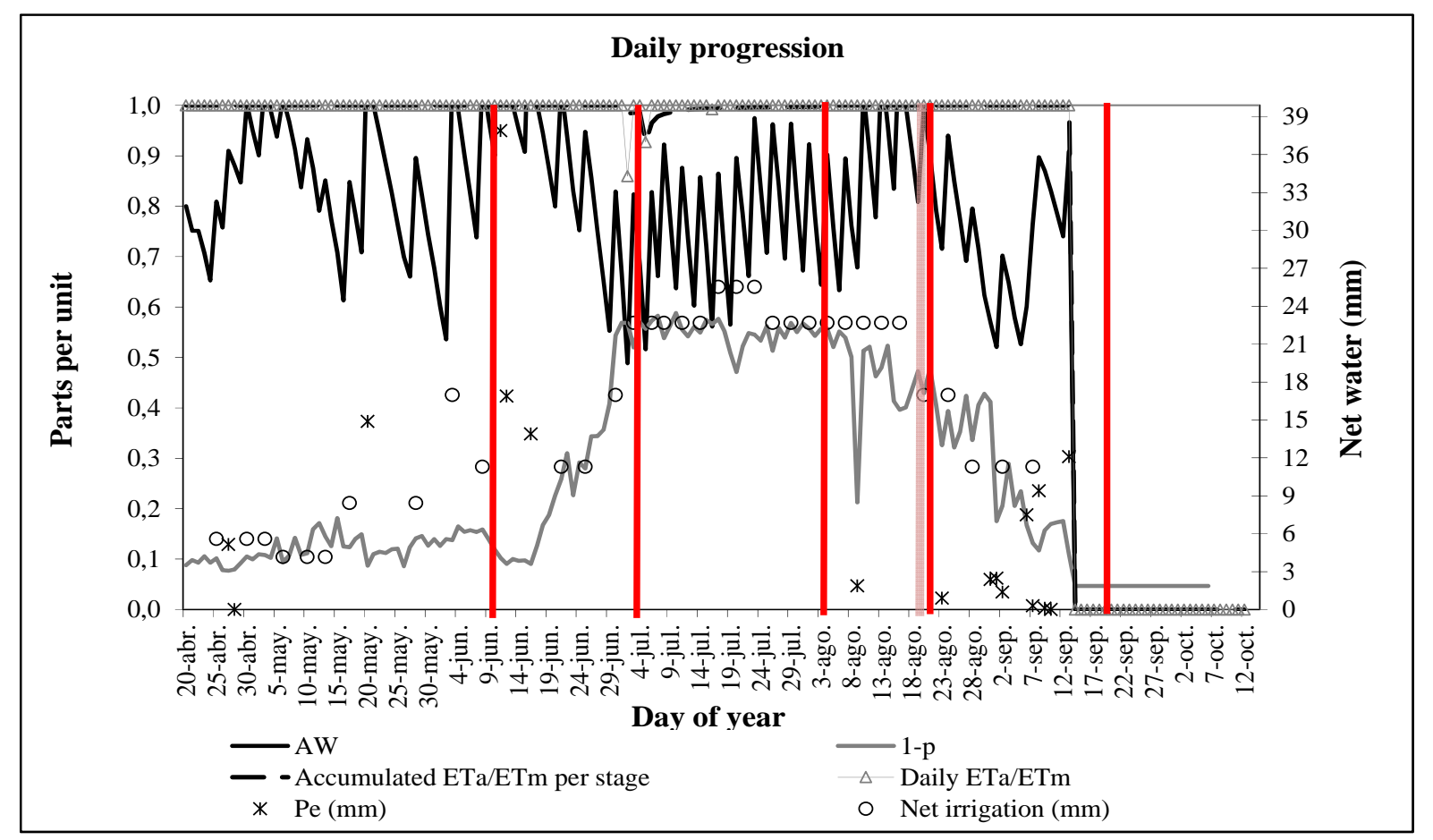

Figura 1. Evolución del agua en el suelo en Maíz Sin Déficit. 


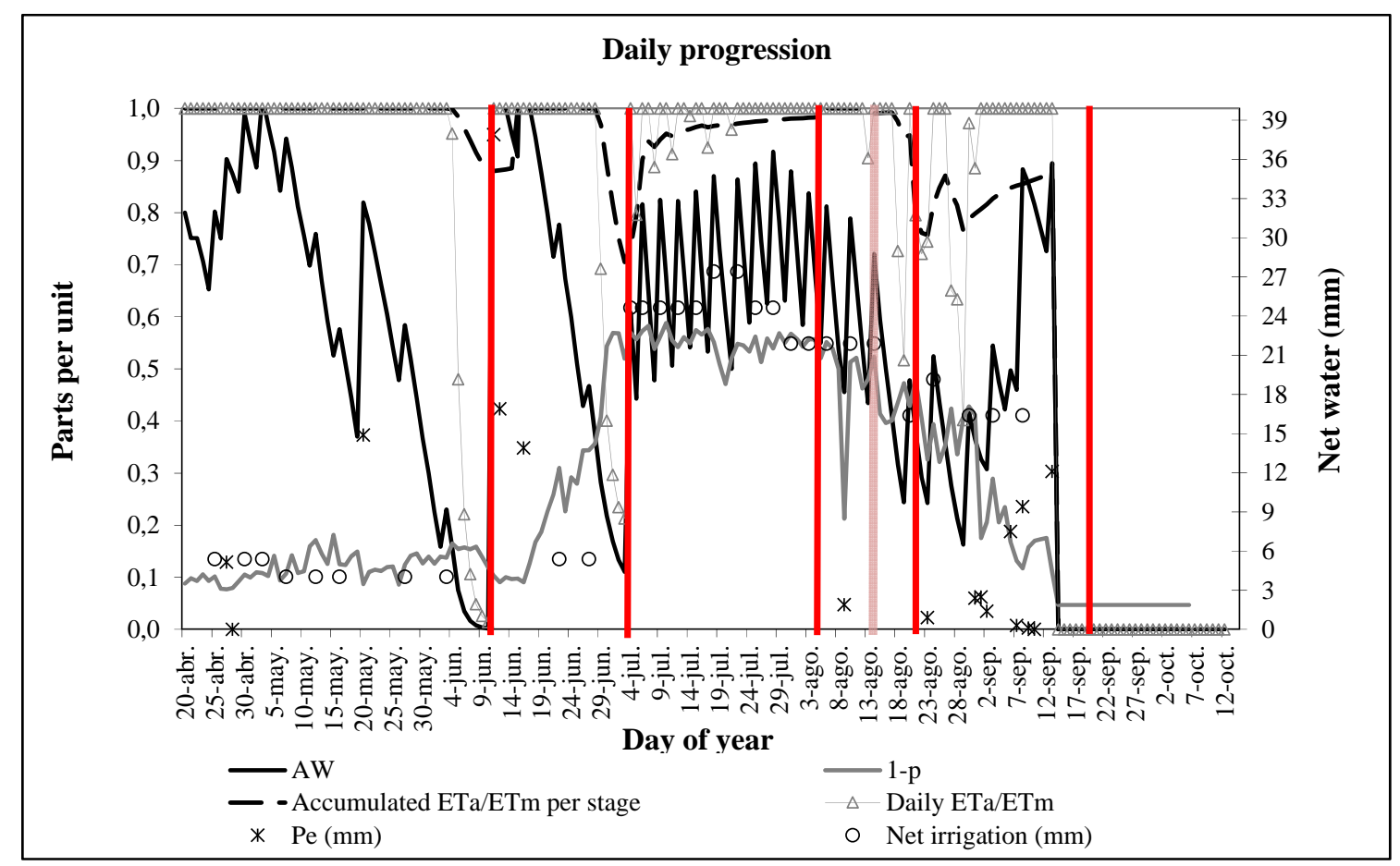

Figura 2. Evolución del agua en el suelo en Maíz ORDI.

Las figuras 3 y 4 muestran la evolución del contenido de humedad del suelo registrado por los sensores volumétricos a 3 profundidades para el caso del maíz.

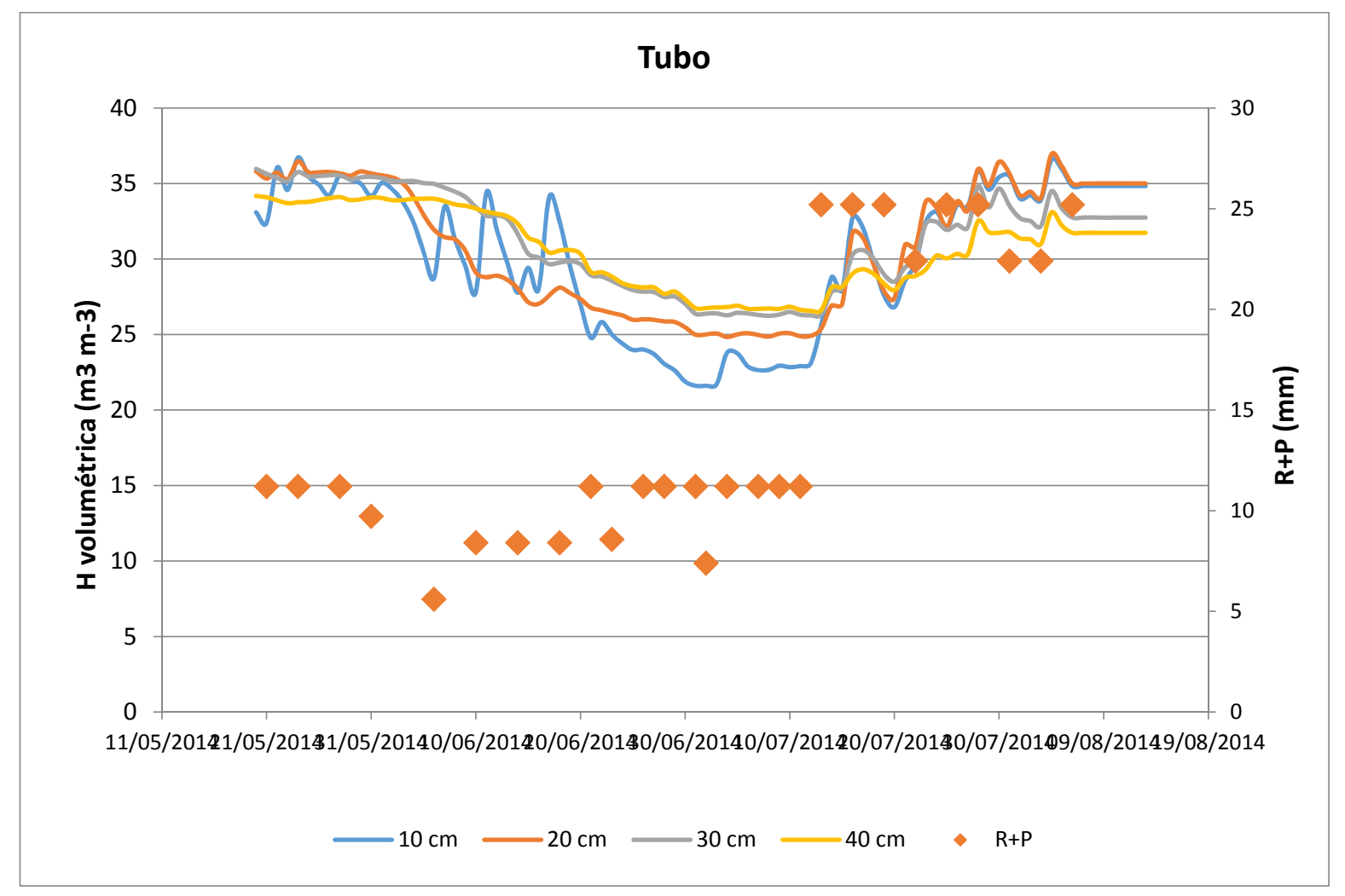

Figura 3. Lectura sonda Enviroscan en Maíz Sin Déficit. 


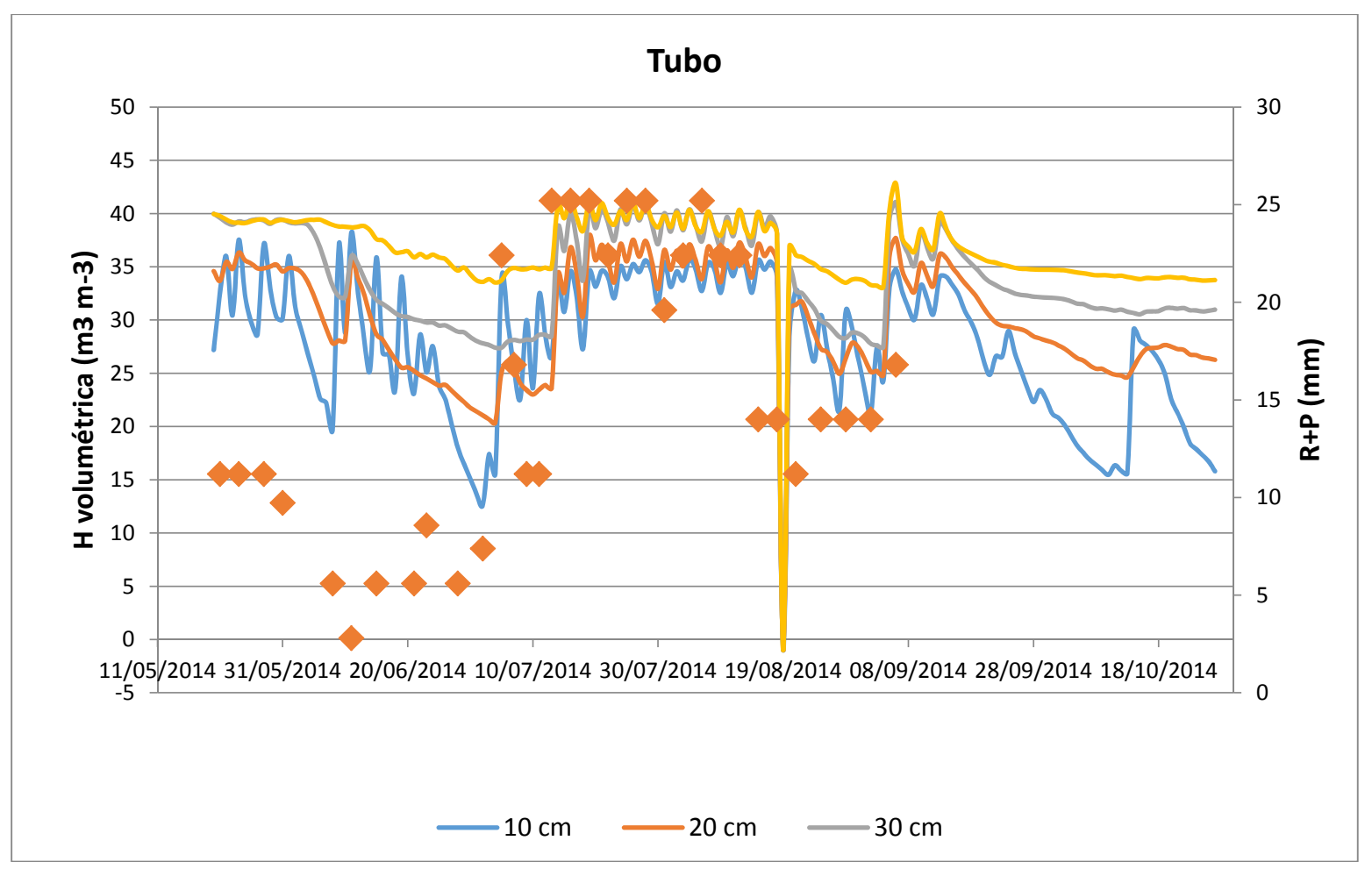

Figura 4. Lecturas sonda Enviroscan en Maíz ORDI.

El modelo MOPECO asignó menores restricciones de agua a los cultivos más rentables como el maíz o la cebolla, es decir, propuso un menor déficit (máximo 10\%). En consecuencia se encontraron menores diferencias de rendimiento neto entre los tratamientos sin restricciones (metodología FAO) y los tratamientos ORDI en estos cultivos (cebolla y maíz) que entre los dos tratamientos de la cebada (Tabla 3). La principal diferencia entre las estrategias es que los tratamientos deficitarios obtuvieron una mayor eficiencia en el uso del agua tanto en términos de rendimiento (EUAY) como en el de margen bruto obtenido (EUAMB) por unidad de volumen de agua de riego aplicada.

Tabla 3. Rendimientos y eficiencias en el uso del agua.

\begin{tabular}{|c|c|c|c|c|c|c|c|c|c|c|}
\hline \multirow[b]{2}{*}{ Cultivo } & \multicolumn{3}{|c|}{ Rendimiento (kg/ha) } & \multicolumn{3}{|c|}{ Agua de riego ( $\left.\mathrm{m}^{3} / \mathrm{ha}\right)$} & \multicolumn{2}{|c|}{$\operatorname{EUAY}\left(\mathrm{kg} / \mathrm{m}^{3}\right)$} & \multicolumn{2}{|c|}{ EUAMB $\left(€ / m^{3}\right)$} \\
\hline & ORDI & $\begin{array}{c}\text { No } \\
\text { déficit }\end{array}$ & $\begin{array}{c}\text { Diferencia } \\
\text { (\%) }\end{array}$ & ORDI & $\begin{array}{c}\text { No } \\
\text { déficit }\end{array}$ & $\begin{array}{c}\text { Diferencia } \\
(\%)\end{array}$ & ORDI & $\begin{array}{c}\text { No } \\
\text { déficit }\end{array}$ & ORDI & $\begin{array}{c}\text { No } \\
\text { déficit }\end{array}$ \\
\hline Ceb & 7479 & 9441 & 26,2 & 2184 & 3220 & 47,4 & 3,42 & 2,93 & 0,30 & 0,29 \\
\hline Cebolla & 91583 & 95646 & 4,4 & 7382 & 7981 & 8,1 & 12,41 & 11,98 & 0,85 & 0,83 \\
\hline Maíz & 14852 & 17360 & 16,9 & 4716 & 5572 & 18,2 & 3,15 & 3,12 & 0,17 & 0,16 \\
\hline
\end{tabular}

La mejora en la eficiencia en el uso del agua permite conseguir una distribución de cultivos que mejora la rentabilidad global de la explotación, en este caso hasta un $11,4 \%$ (Tabla 4). Como era de esperar, el optimizador dedica a los cultivos menos rentables la superficie mínima establecida como restricción. El agua ahorrada con el maíz y la cebada permiten incrementar la superficie de cebolla (también por aplicar a ésta una menor cantidad de agua de riego), pasando de 33,9 ha a 41,8 ha, siendo esta la principal causa de la mejora en la rentabilidad de la explotación. 
Tabla 4. Comparativa económica entre tratamiento sin déficit y ORDI.

Escenario A (Riego sin déficit)

Sup. Mínima por cultivo : 20 ha.

Sup. Total : 100 ha.

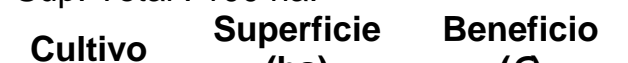

(ha)

Cebada

Cebolla

Maíz

20,0

33,9

20,0

73,9
$(€)$

18.432

225.070

17.575

261.077

\section{Escenario B (Tratamiento ORDI)}

Sup. Mínima por cultivo : 20 ha. Sup. Total : 100 ha.

Cultivo Superficie(ha)

$\begin{array}{lcc}\text { Cebada } & 20,0 & 13.070 \\ \text { Cebolla } & 41,8 & 261.692 \\ \text { Má́z } & 20,0 & 16.053 \\ \text { Total } & \mathbf{8 1 , 8} & \mathbf{2 9 0 . 8 1 5}\end{array}$

Comparativa

Margen Bruto

Beneficio (€/\%)

\section{4- Conclusiones y recomendaciones.}

La combinación de técnicas de riego deficitario optimizado con una adecuada distribución de cultivos, permitiría incrementar el beneficio de las explotaciones para una misma dotación de riego y superficie regable.

Por lo tanto, para una cantidad anual limitada de agua de riego y de superficie cultivable, reducir la aplicación de agua en los cultivos con menor margen bruto para dotar de una mayor superficie y una mayor disponibilidad de agua a los cultivos con mayor margen bruto, hace posible incrementar la rentabilidad económica de las explotaciones de regadío y realizar un uso más eficiente y sostenible de los recursos disponibles, reduciendo adicionalmente la huella hídrica de los productos agrícolas.

\section{5- Bibliografía.}

Allen R.G., Pereira L.S., Raes D., Smith M., 1998. Crop Evapotranspiration: Guide- lines for Computing Crop Water Requirements. Irrigation and Drainage Paper No. 56. FAO, Italy.

Domínguez A., de Juan J.A., Tarjuelo J.M., Martínez R.S., Martínez-Romero A., 2012. Determination of optimal regulated deficit irrigation strategies for maize in a semi-arid environment. Agric. Water Manage., 110, 67-77.

Ortega J.F., de Juan J.A., Martínez-Romero A., Tarjuelo J.M., López-Mata E., 2004. MOPECO: an economic optimization model for irrigation water management. Irrigation Science 23(2), 61-75. 